\title{
Eine Zukunft für Spitäler
}

Beginn einer Artikelreihe, in der eine wesentliche Folge der Einführung von Fallpauschalen im Jahr 2012 beleuchtet wird. Der hier vorliegende erste Teil zeigt die Besonderheit von Spitälern und medizinischen Prozessen auf und warum klassische Optimierungsansätze dieser nicht gerecht werden können. Die weiteren Teile werden sich den Entwicklungen der medizinischen Profession widmen, die Konsequenzen für Spitäler und Prozessoptimierungen aufzeigen und differenzierte Organisationen beispielhaft vorstellen.

Peter Berchtold, Christof Schmitz
Korrespondenz: PD Dr. Peter Berchtold college-M Freiburgstrasse 41 CH-3010 Bern Tel. 0316323026 Fax 0316323025

peter.berchtold@college-m.ch
Mit den DRG-Fallpauschalen soll vieles anders werden in den Schweizer Spitälern ab 2012: mehr Effizienz, kürzere Liegezeiten, bessere Kostentransparenz. Die ersten Erfahrungsberichte fünf Jahre nach der DRG-Einführung in Deutschland beruhigen, da die schlimmsten Befürchtungen nicht eintrafen, und irritieren zugleich: Trotz des Anreizes der Pauschalvergütung finden keine oder kaum Prozessoptimierungen statt. Dafür wird vielerorts über demotivierende Arbeitsverdichtung geklagt, eine bedenkliche Entwicklung in den schon arg gebeutelten Krankenhäusern. Medizinische Prozesse entziehen sich eben weitgehend klassischen Optimierungsbemühungen. Der erste Teil unserer Artikelserie beschäftigt sich mit dieser Besonderheit von Spitälern und geht der Frage nach, welche Optimierungsansätze erfolgreicher sein könnten.

Alfred Koller ist 39-jährig, Agronom und erfolgreicher Bereichsleiter in einem mittelgrossen Unternehmen. Vor 15 Jahren wurde ihm wegen einer Colitis ulcerosa der Dickdarm entfernt, jetzt ist er wegen Gewichtsverlust, Müdigkeit und immer wieder auftretender Gelbsucht in Spitalbehandlung. Auf der Rechnung des Spitals werden später der DRG-Code «H60Z» (Leberzirrhose und bestimmte nichtinfektiöse Hepatitiden mit äusserst schwerer Komorbidität) und ein tiefer fünfstelliger Frankenbetrag stehen. Aber dann wird Herr Koller nach einem turbulenten Spitalaufenthalt längstens wieder zu Hause sein, leider aber in banger Gewissheit, dass viele weitere Abklärungen und Behandlungen vor ihm liegen, vielleicht sogar eine Lebertransplantation in nicht allzu ferner Zukunft. Er wird in den folgenden Jahren viele Ärzte, Pflegende und anderes Spitalspersonal kennenlernen und beobachten können, wie sich ein Netzwerk von Spezialisten um ihn und für ihn gruppiert. Wird er staunen können, von welch intensiver $\mathrm{Zu}$ sammenarbeit er profitieren kann? Oder wird er feststellen müssen, wie gering die wechselseitige Bezugnahme der verschiedenen Beteiligten ist? Und: Wie wenig bzw. wie sehr wird er bemerken, dass vergütungstechnische Fragen seine Behandlung beeinflussen?

\section{Patientenzentrierte Spitalarbeit nach 2012?}

Sie werden wohl eingeführt, die DRG-Fallpauschalen (Diagnosis Related Groups), und damit wird in den Schweizer Spitälern ab 2012 vieles anders: vergütet wird fortan nicht mehr nach Aufwand oder per Tagespauschale, sondern mit einer diagnosespezifischen (DRG-)Fallpauschale für den gesamten stationären Aufenthalt. Damit sollen die Spitäler (noch) stärker unter Effizienzdruck gesetzt werden, beispielsweise, damit sie die Verweildauer der Patientinnen und Patienten verkürzen. Durch die landesweite Einführung der DRG-Vergütung wird darüber hinaus eine bessere Kostentransparenz ermöglicht, die ihrerseits den Wettbewerbsdruck auf die Spitäler erhöht. Diesen Druck werden die behandelnden Ärzte und Pflegenden vor allem als Ökonomisierung der klinischen Arbeit erfahren, die - so eine häufige Sorge - eine auf die Bedürfnisse der Patienten ausgerichtete Behandlung nicht mehr zulässt. Das ist genau das, was mindestens in anekdotischer Form oder ersten Resultaten von DRG-Begleitstudien aus Ländern, die bereits seit einiger Zeit mit DRGs arbeiten, immer wieder zu hören ist: vorzeitige Entlassungen aus der Spitalbehandlung bei (noch) fehlenden Nachsorgeangeboten, keine ganzheitliche Betrachtung der Patienten vor allem bei komplexen Erkrankungen, neue Fragmentierungen der Behandlungsketten usw.

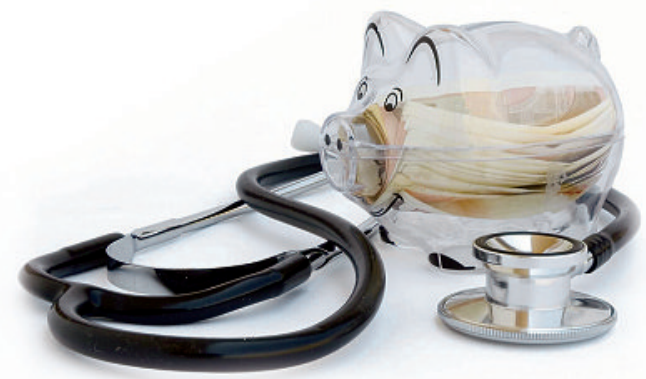

Fallpauschalen sind der Versuch, Nichtmedizinisches mit Medizinischem zu verbinden. 
Die Datenlage zur Entwicklung der Versorgungsqualität nach DRG-Einführungen ist uneindeutig. Vieles spricht dafür, dass sowohl die grossen Befürchtungen als auch die grossen Hoffnungen nicht eintreffen: also keine «blutigen Entlassungen», aber auch keine echten Kostensenkungen. Unterhalb der Ebene von Globalmessungen aber finden sich viele Veränderungen, die der groben Optik systemweiter For-

\section{Ein neues Vergütungs- (und Anreizsystem) wird ein- geführt, und eine der zentralen Annahmen geht fehl}

schungsansätze verborgen bleiben und trotzdem die konkreten Patienten (und das medizinische Personal) betreffen. Deshalb stellt sich die Frage in aller Schärfe: Welche Folgen werden die DRG-bedingten Änderungen für Herrn Koller haben? Negative, weil z. B. gerade Patienten wie er, chronisch krank, multimorbid, wenig in diese neue Vergütungswelt passen? Oder gar positive, weil Prozesse optimiert werden?

Für eine mögliche Antwort auf diese Frage ist ein besonderes Ergebnis der DRG-Begleitforschung aus Deutschland relevant. Eine zentrale (betriebswirtschaftlich begründete) Annahme jeder DRG-Einfüh-

Abb. 1

Das Kreuz des Spitals.

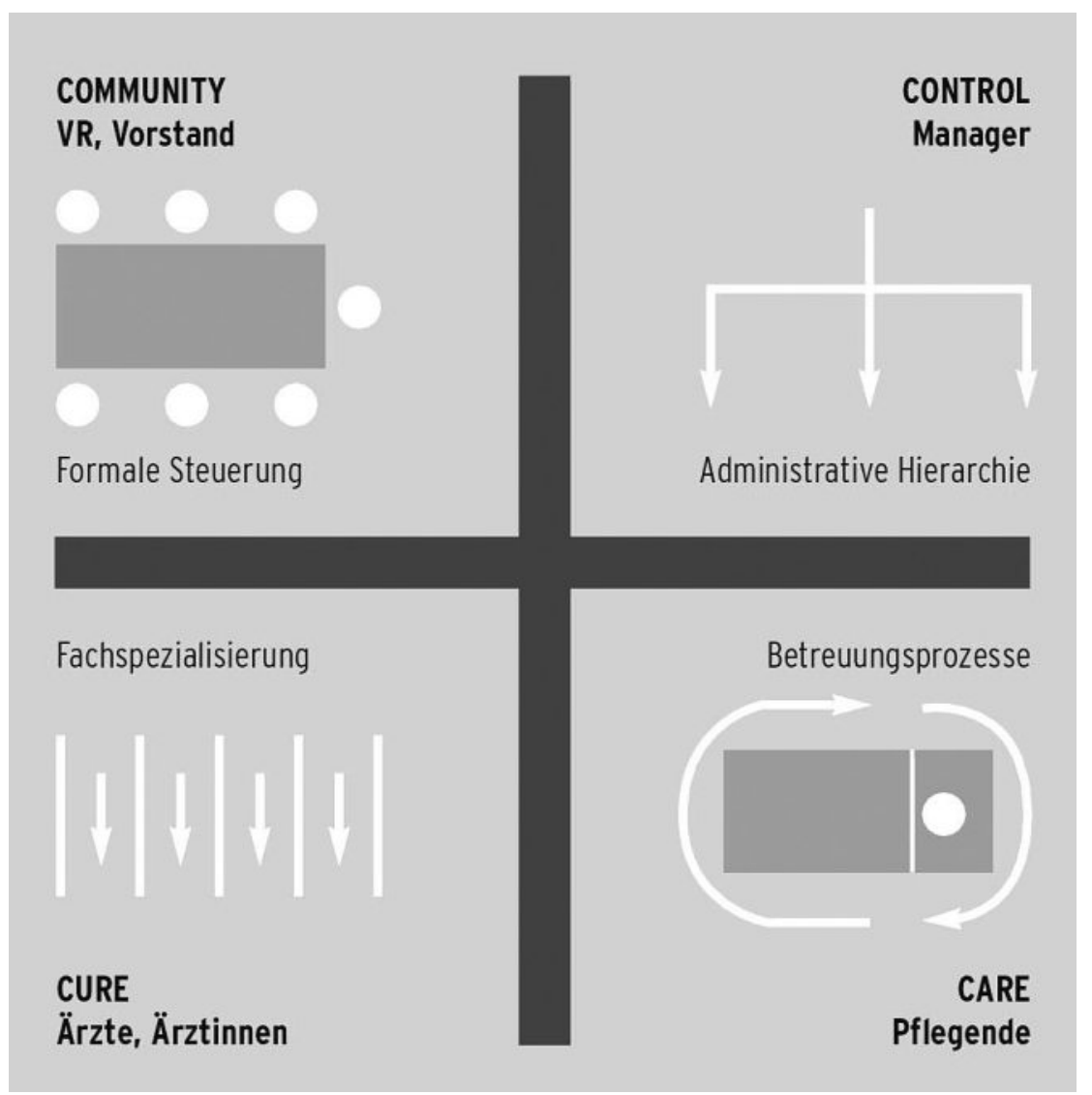

rung ist, dass dieses Vergütungssystem Spitäler dazu anhält, Prozesse zu optimieren und dadurch Verweildauern zu verkürzen und ineffektive Leistungen abzubauen. Bemerkenswert ist nun, dass in Deutschland zwar die Verweildauer - der Annahme entsprechend - zurückgeht, aber die Prozesse - entgegen der Annahme - nicht oder nur beschränkt adaptiert werden. Berichtet werden hingegen Arbeitsverdichtungen, Unzufriedenheit und Demotivation der medizinischen Kader [1, 2]. Aus Sicht dieser Ärzte kann nicht mehr eine in ihrem Sinne angemessene «gute Medizin» geleistet werden [3]. Für alle Organisationen gilt es eigentlich als Warnsignal, wenn die oberen Kader mit Motivationsproblemen zu kämpfen haben.

\section{Sind Prozesse unverbesserbar?}

Das ist eine starke Sache: Ein neues Vergütungs- (und Anreizsystem) wird eingeführt und eine der zentralen Annahmen, die zur Einführung motivieren, geht fehl. Die Evidenz, dass dem so ist, ist bei aller vorhandenen Uneindeutigkeit stark genug, um sich mit der Frage intensiver zu beschäftigen, was das Spezielle an den medizinischen Prozessen bzw. an den Spitälern ist. Und warum, allen ökonomischen Anreizen zum Trotz, etablierte Praktiken sogar unter Zahlung zusätzlicher Preise durch das medizinische Personal (Stichwort «Arbeitsverdichtung») bestehen bleiben.

Die Medizin ist eine besondere Art «Geschäft», eines, für das Gesellschaften die Sonderform der Profession erfunden haben. Professionen sind dort relevant, wo es um die Bewältigung von «Krisenlagen» geht, in diesem Fall um Krisen der somato-psychosozialen Integrität. Bei Krisen dieser Art kann nicht wie beim Ingenieur präzise berechnet werden, wie die Brücke halten wird, sondern gilt es, Interventionen in lebendige, unberechenbare (!) Systeme zu tätigen. «In medicine, uncertainty is the water we swim in» [4]. Das ist die Grundthematik, um die es geht: Berechenbarkeit vs. Unberechenbarkeit, Routine vs. Krise [5].

In diesem «Krisen-Geschäft», in der klinischen Arbeit, geht es im Kern um den Umgang mit Ungewissheit. Eine Ungewissheit, die darin besteht, dass nur begrenzt vorhergesehen - «berechnet» - werden kann, welchen genauen Verlauf eine konkrete Krankengeschichte nehmen wird [6]. Konkret vorhergesehen werden können Verläufe nur dort, wo Ursache und Wirkung klar zuordenbar sind (z. B. Insulinmangel bei Diabetes), weder Komorbiditäten noch Nebenwirkungen auftauchen und der Patient sich entsprechend verhält. Dort funktionieren Guidelines, Behandlungspfade u. Ä. Wenn Ungewissheit über Verläufe existiert, entziehen sich Prozesse klassischen Optimierungsbemühungen, die im Grundsatz Standardisierungen von Vorhersehbarem intendieren. Der Umgang mit Ungewissheit ist zentraler Orientierungspunkt für jede Ärztin und jeden Arzt. Sie lernen früh zwischen Lehrbuchwissen und konkreten Patienten und zwischen wissenschaftlicher Legitimation und «Kunst des Arztes» hin- und herzuspringen. 
Von daher sind individualistische Konzeptionen der klinischen Arbeit - Motto: Jeder Patient ist anders und darauf müssen wir vorbereitet sein - tief verwurzelt und sorgen für Qualität [7].

Individualistische Konzeptionen der klinischen Arbeit vertragen sich schlecht mit Systematisierung und Standardisierung. Das ist der eigentliche Streitpunkt, um den es bei der Einführung der DRG-Vergütung geht. DRGs sind Versuche, Systematisierungen vorzunehmen und Nichtmedizinisches (u.a. Ökonomie) mit Medizinischem zu verbinden. Eine der Grundideen der DRGs ist ja: Wenn 40 Spitäler durchschnittlich so und so lange für eine spezielle Diagnose benötigen, dann soll dieser Durchschnitt nun (mit einer gewissen Bandbreite an Abweichungsmöglichkeiten) geltend sein.

\section{Individualistische Konzeptionen der klinischen \\ Arbeit vertragen sich schlecht mit Systematisierung und Standardisierung}

Dieser Streitpunkt betrifft aber auch die Organisation selbst, das Spital, das die Aufgabe hat, professionelles Handeln zur Verfügung zu stellen, zu entwickeln und zu steuern - zu managen. Spitäler unterscheiden sich von anderen Organisationen durch ihre aussergewöhnlich starke interne Differenzierung mindestens vierer Subsysteme mit je eigenen Kulturen, Verständnissen und Logiken (s. Abb. 1) [8]. Wir haben diese Besonderheit das «Kreuz des Spitals» genannt und an verschiedenen Stellen ausgeführt [9]. Besonders relevant ist der horizontale Balken, die sog. «clincal divide». Der Balken zwischen den beiden kerngeschäftfremden Subsystemen «oben» und den beiden kerngeschäftnahen «unten» bedeutet eine starke Barriere und macht erfolgreiches Management von Spitälern so herausfordernd. Dazu passt die Beobachtung, dass sich in vielen Spitälern die Einschätzungen zu den Folgen der DRG-Einführung stark unterscheiden, je nachdem ob man «oben» oder «unten» als Gesprächspartner hat. Während etwa die Geschäftsleitung «oben» beschreibt, dass ein Entlassungsmanagement eingeführt wurde, scheinen die Ärzte «unten» nichts davon zu wissen.

\section{Chancen}

Die Art und Weise, wie Patientenversorgung in Spitälern und Gesundheitswesen organisiert ist, war bis anhin stark durch die medizinische Profession geprägt, sowohl hinsichtlich der Art der Krankenbehandlung als auch der Werte, Regeln und Beziehungen, die konkret gelten. Vor allem die Profession hat bis jetzt entschieden, wie Kranke behandelt werden. Die sogenannte Ökonomisierung der Medizin ist ein möglicher Wendepunkt. Nicht mehr Medizinisches alleine entscheidet, auch anderes - Fachfremdes - soll (stärker als bis anhin) einbezogen werden. Diese Veränderung ist sicherlich teilweise schmerzlich, sie birgt aber auch Chancen. Noch bleibt offen, ob Herr Koller 2014 immer noch engagierten oder demotiviert wirkenden Ärzten gegenübersitzen wird und ob er staunen muss, wie wenig das, was die verschiedenen Spezialisten sagen, zusammenpasst oder er beeindruckt sein wird, von welch konzertierter Aktion er profitierten kann. Unsere Hypothese ist, dass Letzteres dann eintreten wird, wenn Entwicklungen, die aus der Medizin selbst stammen, genutzt werden - und nicht bloss äussere Impulse «abgefedert» werden müssen. Wir wollen daher in den weiteren Artikeln dieser Reihe aufzeigen und eine Diskussion darüber lancieren, welche Möglichkeiten durch eine sich implizit bereits entfaltende Differenzierung der Spitalmedizin vorhanden sind und wie sich die darin liegenden Potentiale freilegen lassen.

Der nächste Teil der Reihe ist für Ausgabe 50 vom 15. Dezember 2010 geplant.

\section{Literatur}

1 Brügger U. Impact of DRGs - Introducing a DRG reimbursement system: A literature review. SGGP Schriftenreihe. Band 98; 2010.

2 Klinke S. Auswirkungen des DRG-Entgeltsystems auf Arbeitsbedingungen und berufliches Selbstverständnis von Ärzten und die Versorgungsqualität in deutschen Krankenhäusern. Teil II. Wissenschaftszentrum Berlin für Sozialforschung (WZB).

3 Vogd W. Die Organisation Krankenhaus im Wandel. Studien zur Gesundheits- und Pflegewissenschaft. Bern: Hans Huber; 2006.

4 Sanders L. Every Patient tells a Story. New York: Crown; 2009.

5 Oevermann U. Wissenschaft als Beruf. Die Professionalisierung wissenschaftlichen Handelns und die gegenwärtige Universitätsentwicklung. In: Die Hochschule - Journal für Wissenschaft und Bildung. 2005;14(1):15-40.

6 Bohmer R. Designing Care. Harvard Business Press; 2009.

7 Degeling P, Maxwell S, Kennedy J Coyle B. Medicine, management, and modernisation: A dance macabre? Brit Med J. 2003;326(7390):649-652.

8 Glouberman S, Mintzberg H. Managing the care of health and the cure of disease - Part I: Differentiation. Health Care Manage Rev. 2001;26(1):56-92.

9 Berchtold P, Schmitz C. Managing Professionals. Amelung V. et al (Hrsg.). Vernetzung im Gesundheitswesen. Stuttgart: Kohlhammer; 2009. 